\title{
EL CONCEPTO DE VALENCIA: SU CONSTRUCCIÓN HISTÓRICA Y EPISTEMOLÓGICA Y LA IMPORTANCIA DE SU INCLUSIÓN EN LA ENSEÑANZA
}

\author{
The valence concept: its historical and epistemological \\ construction and its importance to teaching.
}

\author{
Rómulo Gallego Badillo \\ Royman Pérez Miranda ${ }^{2}$ \\ María V. Uribe Beltrán ${ }^{3}$ \\ Luigi Cuéllar Fernandezt \\ Rafael Y. Amador Rodríguez
}

Resumen: El objetivo de éste escrito es reconocer la importancia de la historia y la filosofía de los modelos, teorías y conceptos científicos en la enseñanza de las ciencias, en particular en la enseñanza del concepto de valencia, además de la forma como es presentado éste en los libros de texto. El estudio histórico y epistemológico de este concepto fue realizado desde los criterios establecidos por los autores, aplicado a algunos de los libros de texto utilizados en educación básica y media del sistema educativo Colombiano.

Palabras claves: Historia y filosofía de las ciencias, valencia, enseñanza y libros de texto.

Abstract: The aim of this article is to promote a better understanding about the important role played by both the history and the philosophy of the science on the teaching of the scientific concepts, theories and models, in particular on the teaching of "the valency" concept; and, how is it presented in textbooks. Thus, a historic and epistemological study of this concept was done in order to define the main criteria to analyze different textbooks that are used at both secondary and university levels of the education in the Colombian system.

Keywords: History and epistemology of science, valence, teaching and text books.

\section{Introducción}

En la actualidad, según el consenso de los investigadores en didáctica de las ciencias, se ha reconocido la importancia de la inclusión de la historia de las ciencias en su enseñanza, de conformidad con los resultados obtenidos a partir del análisis de la transposición didáctica de algunos conceptos científicos (Lombardi, 1997). Sin embargo, como señala el autor citado, hay quienes manifiestan que es preferible prescindir totalmente de la historia, ante la imposibilidad de conocer todos los elementos involucrados en la historia misma de la formulación de un concepto, lo que llevaría a hacer una "casi historia" o "mala historia" o a presentar una reacomodación de algunos factores de ésta, según sus compromisos e intereses, que terminaría siendo una historia simplificada y recortada.

\footnotetext{
${ }^{1}$ Docente y Director del Grupo IREC. Universidad Pedagógica Nacional. (e-mail: rgallego@uni.pedagogica.edu.co)

${ }^{2}$ Docente y Codirector del Grupo IREC. Universidad Pedagógica Nacional. (e-mail: royman@uni.pedagogica.edu.co)

${ }^{3}$ Maestría en Docencia de la Química, Grupo IREC. Docente Universidad de La Sabana. (e-mail: mariav.uribe@unisabana.edu.co)

${ }^{4}$ Maestría en Docencia de la Química, Grupo IREC. Docente Instituto San Bernardo de La Salle, Docente Universidad Pedagógica Nacional. (e-mail: luigi_cuellar@uni.pedagogica.edu.co)

${ }^{5}$ Maestría en Docencia de la Química, Grupo IREC. Docente Fundación Universitaria Manuela Beltrán.

(e-mail: rafael_amador@pedagogica.edu.co)
} 
Los autores del presente artículo, y de acuerdo con Lombardi (1997), sin desconocer las dificultades que pueden presentarse en la enseñanza de las ciencias, son del parecer de que la legitimidad de la revisión de la historia relacionada con la formulación de un saber determinado no se basa en el carácter completo de los datos recogidos, pues tal "completitud" teórica es difícil de alcanzar. Ante ello consideran que la introducción de esta disciplina en la enseñanza de las ciencias permite seleccionar y organizar tal información, con el fin de establecer un hilo conductor que dé cuenta de la historia interna y externa (Lakatos, 1983), en el que los hechos reconocidos encuentren sentido, lo que facilita una permanente reelaboración de las interpretaciones producto de las reflexiones históricas.

En tal selección, es importante no caer en un reduccionismo de la actividad científica, al cual se llegaría si se hiciera un "recuento historiográfico" en el que se presenten, únicamente, aquellas construcciones catalogadas como relevantes para la consolidación del pensamiento científico ulterior, lo cual llevaría a una visión distorsionada de la historia de las ciencias como una mera narración de acontecimientos.

Es importante, para combatir tal imagen distorsionada de la ciencia y de la actividad científica, incorporar el estudio histórico con el fin de que estas se presenten como un proceso de investigación (Solves y Traver, 1996), seleccionando aquellos contenidos estructurantes que permitan al estudiante abordar problemas significativos, generando ámbitos pedagógicos y didácticos, planteando situaciones que posibiliten, por parte de dichos estudiantes, la reconstrucción permanente de sus estructuras conceptuales y metodológicas relacionadas con los conocimientos científicos.

Cabe señalar, como se desarrollará más adelante, que en Colombia los aspectos de tipo histórico se encuentran ausentes en la mayoría de los libros de texto y que, cuando aparecen son tratados en forma superficial, sin atribuirles un papel relevante, igual a como ocurre en otros países (Solves y Traver, 1996); esto sumado al papel del desempeño del profesor como instrumento transmisor acrítico de los contenidos curriculares, es causa del desinterés de los alumnos por el estudio de las ciencias.

Sin embargo, no puede olvidarse que el objetivo de los trabajos de investigación de los didactas de las ciencias de la naturaleza no se limita al campo de la historia de las ciencias, por lo cual el estudio de esta última no pretende establecer "verdades" históricas, sino aprovechar los aportes de la investigación en la historia de las ciencias para mejorar su imagen y su enseñanza (Solves y Traver, 2001).

Se considera que la historia del concepto de valencia se inicia con las leyes de las proporciones definidas y múltiples, a partir de las cuales Thomas Thomson (1813) pudo concluir que cada elemento tiene un número característico de puntos de unión, coincidiendo con lo que, 40 años después, propuso Edward Frankland (1852) al decir que cada elemento tiene una "atomicidad" definida, considerada ésta como la capacidad de combinación de los átomos de cada elemento. Posteriormente, y gracias a la proposición de los modelos atómicos, se estableció la relación entre los electrones más externos del átomo y la capacidad de combinación de los mismos, y se propuso como valencia de un átomo el número de electrones de su capa más externa. Este fue el concepto de valencia que utilizaron Lewis (1916) y Langmuir (1919) para desarrollar sus teorías del enlace químico, y que permaneció hasta cuando Linus Pauling (1932) lo explicó desde la mecánica cuántica. 


\section{Importancia de la Historia y la Filosofía de la Ciencia en la enseñanza de las Ciencias}

Aducen los investigadores en Didáctica de las Ciencias, y en especial aquellos que se han preocupado por la relación inseparable entre la historia de las ciencias y la enseñanza de las mismas, que en el trabajo docente habitual se desconoce la importancia de la historia, lo que lleva a que sea considerada como una empresa individual y absolutista, que muestra una única verdad (Solbes y Traver, 1996). La enseñanza de una ciencia ha de considerar la evolución de la misma, ya que de no ser así el docente transmitiría una imagen deformada de la actividad científica (Solbes y Traver, 2001), esto es, que los estudiantes, bien sean de educación básica, media o superior, estarían construyendo y reconstruyendo un concepto desfigurado de ciencia, producto de una mirada empiropositivista (Gallego Badillo y Pérez Miranda, 1999).

Por el contrario, cuando el profesor incluye la historia de las ciencias en su ejercicio docente, además de conocer la materia que enseña (Gil, 1991), reconoce los problemas que se originaron en la construcción del conocimiento científico, las implicaciones que tuvo en la comunidad científica la formulación de las teorías o modelos, los problemas que estos solucionaron y aquellos que dejaron de lado. Así se pone de manifiesto que la ciencia es una construcción comunitaria (Hodson, 1985) que no sigue un proceso lineal y acumulativo.

Uno de los elementos socializadores del conocimiento científico en la escuela es el libro de texto y, tradicionalmente, su utilización se reduce a que el lector encuentre en éste información que puede ser considerada como conocimiento acabado o absoluto; esto ocurre debido a que si, quien lee no llega a conocer la evolución del concepto científico que estudia, no puede llegar a entender que éste puede ser objeto de posteriores reformulaciones. Por el contrario, el reconocimiento de los hechos que han motivado la construcción y reconstrucción de conceptos, teorías y modelos científicos llevan a identificar la actividad científica como una empresa dinámica.

La vinculación de la historia de las ciencias en su enseñanza, en los niveles educativos básico y medio, muestra a los niños y jóvenes que la construcción científica no es llevada a cabo por individuos especiales y aislados del resto de la sociedad, que tienen aptitudes innatas para la ciencia, sino que por el contrario, los hombres y mujeres de ciencias que se han dedicado a la elaboración de esta, son personas comunes, con problemas e ilusiones; lo que los diferencia de los demás es la dedicación, constancia e interés por construir y aportar en la edificación de la ciencia. Además, propicia en el estudiantado el interés por preguntarse por las situaciones problemáticas que se suscitaron en la formulación de las teorías o modelos científicos.

En los párrafos anteriores se ha hecho alusión a la historia interna de las ciencias (Lakatos, 1983); sin embargo, al abordar la enseñanza de las ciencias en estos niveles educativos, no se debe olvidar su historia externa relacionada con el contexto en el cual se desarrolla una teoría o modelo, puesto que la construcción de la ciencia como tal tiene implicaciones sociales, culturales, políticas y económicas que le permiten, o no, su evolución.

La inserción de la historia en la enseñanza de las ciencias permite a los estudiantes realizar la construcción y reconstrucción de sus estructuras conceptuales, metodológicas, actitudinales y axiológicas (Gallego, Pérez, Torres de G. y Amador, 2004), teniendo presente que el propósito, en estos niveles de educación básico y medio, es educar en ciencias y no formar científicos, dado que esto le concierne a las instituciones de educación superior, especialmente a los programas de formación inicial y continua de profesores de ciencias, en los que adquiere relevancia puesto que de esto depende la versión de ciencia que enseñen. En uno de los casos se haría desde unos presupuestos empiropositivistas (Bacon, 1620), y en el otro se puede enseñar ciencias desde presupuestos constructivistas, teniendo en cuenta los siguientes planteamientos epistemológicos: 
A partir de las revoluciones epistemológicas acontecidas en los primeros años del siglo pasado, los hombres y mujeres de ciencias se dieron a la tarea de preguntarse por la naturaleza de las ciencias y del desarrollo del conocimiento científico. Desde el constructivismo, como versión epistemológica, se puede proponer que el conocimiento se ha desarrollado desde lo planteado por K. Popper (1962), quien hace una crítica a la lógica inductiva, refutando que la construcción y desarrollo del conocimiento científico no se puede afrontar desde dicha lógica, por lo cual propone trabajar bajo la lógica deductiva, formulando que la categoría que da cuenta del desarrollo y construcción del conocimiento es la de teoría, y que la tarea de los hombres y mujeres de ciencias es formular proposiciones y sistemas de proposiciones que han de ser contrastadas rigurosamente.

Desde la misma lógica deductivista, T. Kuhn (1972) propone que el desarrollo y construcción del conocimiento científico obedece a la categoría de paradigma, de paradigmas en competencia, de crisis paradigmática y de revolución científica.

Otra versión es la que propone I. Lakatos (1983), quien realiza una crítica a las propuestas de Popper y Kuhn, formulando la categoría de programa de investigación científica, el cual está constituido por un núcleo firme conformado por las leyes o proposiciones centrales que direccionan el programa de investigación y por un cinturón protector, el cual está conformado por hipótesis auxiliares que reciben las contrastaciones empíricas. Señaló cuáles deben ser los pasos para la confrontación de un programa científico de investigación: Inicialmente se estudia si los hechos conocidos o las predicciones están de acuerdo con el "heurístico positivo". De ser así se refuerza el núcleo central; en caso contrario se modifica el cinturón protector o "heurístico negativo" sin afectar al núcleo. En aquellos casos en que, a pesar de estas últimas modificaciones, las teorías continúen sin dar explicación a problemas, el programa está seriamente amenazado y puede llegar a ser remplazado por otro. Con esto define dos tipos de programas de investigación: los de carácter progresivo y los de carácter regresivo, a los cuales llamó "degenerados". Los primeros son aquellos cuyo crecimiento teórico permite que se puedan seguir prediciendo hechos exitosamente y que serán los que pueden llegar a remplazar a aquellos con carácter regresivo, cuyo desarrollo teórico está estancado.

Otro concepto que está cobrando fuerza para explicar el desarrollo histórico de las ciencias es el de modelo. Los autores del presente artículo son del parecer de que los modelos científicos son representaciones provisorias y perfectibles del objeto o fenómeno en estudio, aceptadas por la comunidad de científicos, que muestran algunos aspectos de estos, de acuerdo con su intención (Islas, S.M. y Pesa, M., 2003). Los modelos científicos se conectan con la realidad por medio de analogías que se expresan en hipótesis que, una vez contrastadas, serán teorías. En otras palabras, los modelos científicos son representaciones teóricas de sistemas de teorías que, a su vez, son representaciones simplificadas e idealizadas de la realidad y que se conectan con esta última por medio de analogías susceptibles de ser contrastadas empíricamente (Adúriz-Bravo, 2001).

Lo anterior es el marco conceptual desde el cual se propone enseñar las ciencias, para cumplir con el propósito de que los estudiantes den cuenta de la construcción de las ciencias experimentales, ya que se establece que tal conocimiento ha de ser construido por ellos, especialmente por aquellos que pertenecen a programas de formación inicial y continua de profesores de ciencias (Adúriz-Bravo, 2001).

\section{Construcción histórica y epistemológica del concepto de valencia}

Desde el momento en el que se pensó que algunas de las partículas que componían las sustancias estaban conformadas por átomos unidos, empezó a existir inquietud sobre la manera 
como los átomos se unían para formar compuestos y la capacidad de enlazarse los átomos de cada elemento. Se ha de considerar las leyes de las proporciones definidas y múltiples como la base para la construcción histórica del concepto de enlace químico y, además, del concepto de valencia, íntimamente relacionado con el primero, pues se refiere al número de enlaces que un elemento puede formar con átomos de otros elementos.

John Dalton, a principios del siglo XIX, describió los átomos como partículas materiales indivisibles que tenían unos puntos de unión, por medio de los cuales se ligaban, unos con otros, para formar todos los compuestos presentes en la naturaleza. De acuerdo con su teoría atómica, los puntos de unión variaban considerablemente de un elemento a otro, siendo esto característico de los átomos de cada elemento. Los átomos de hidrógeno parecían tener un solo punto de unión, mientras que los de oxígeno parecían tener dos, usualmente, y los del carbono cuatro. Algunos elementos tenían una capacidad variable para unirse, dependiente del otro elemento con el cual se uniera (Thomson T., 1813).

$\mathrm{Al}$ respecto, Thomas Thomson (1813), quien se refirió a la teoría de las proporciones definidas como construcción de J. Dalton aunque, realmente la contrucción teórica se debió a J.L. Proust, la considera el más grande paso que la química ha dado en su desarrollo como ciencia. Thomson hizo alusión a algunos experimentos, elaborados y realizados por Berzelius, con los cuales confirmó la doctrina de Dalton y dedujo leyes para determinar la constitución de los cuerpos. También, se propuso exponer la naturaleza de la teoría de Dalton y sus fundamentos, además de elaborar una tabla que mostrara las proporciones numéricas en las cuales se combinan las sustancias, deducidas de la aplicación de la ley Daltoniana a los resultados de los análisis hechos. Se refirió a los átomos de los elementos como puntos físicos indivisibles rodeados de esferas de atracción y repulsión, que se unen para formar compuestos, que contienen proporciones constantes de sus constituyentes (el agua es universalmente compuesta por una parte de hidrógeno y 7,5 partes de oxígeno, por peso). Dijo que la única manera de explicar esta permanencia de los compuestos químicos se debía, exclusivamente, a que un número determinado de los átomos de un elemento se unían con un número determinado de los átomos del otro elemento.

Edward Frankland (1852) desarrolló el concepto de atomicidad, que equivale a lo que hoy se conoce como valencia, investigando sobre las propiedades de compuestos organometálicos. Sugirió que cada elemento formaba compuestos con cantidades definidas de otros elementos, ya que cada uno tiene una "atomicidad" fija, considerando esta como la capacidad de combinación de los átomos de cada elemento, en comparación con la del hidrógeno que siempre es una. Es importante anotar que, en este artículo, Frankland nombró como elementos electro-negativos al cloro, bromo y yodo, así como al etilo y otros radicales (a cuyas partículas nombraba como átomos) y como electro-positivos a los elementos metálicos, aunque no esbozó ninguna definición de estos términos. En el mismo artículo, Frankland concluyó, de los resultados de sus experimentos, que el nitrógeno, el fósforo, el antimonio y el arsénico exhiben la tendencia a formar compuestos que contienen 3 y 5 equivalentes de otros elementos, es decir, presentan dos números de atomicidad diferentes. Los trabajos y teorías de Frankland sirvieron de base para la tabla periódica que propuso Mendeleev, en la cual relacionó la valencia de los elementos con su posición en la tabla.

Friedrich Kekule (1865) propuso la estructura cíclica del benceno, basándose en la teoría de la atomicidad de los elementos de Frankland y en el conocimiento, construido en trabajos propios anteriores, del carbono como elemento tetratómico; pudo explicar la estructura de muchos compuestos del carbono, particularmente de aquellos a los que llamaba cuerpos grasos (conocidos hoy como compuestos alifáticos). Estaba seguro de poder aplicar estos mismos principios a los compuestos aromáticos, a pesar de que había algunos científicos que 


\section{Rómulo Gallego Badillo, Royman Pérez Miranda, María V. Uribe Beltrán, Luigi}

Cuéllar Fernández y Rafael Y. Amador Rodríguez

habían expresado que la composición de estos compuestos no se podía explicar por la teoría de la atomicidad e, incluso, que existía un grupo hexatómico formado por seis átomos de carbono, sin interesarles cómo se unían estos. En este artículo (Kekule, 1865) se continúa llamando atomicidad a lo que poco más adelante se conoció como valencia, de tal manera que nombró al átomo de carbono tetratómico (luego tetravalente) y constitución atomística de los compuestos (a lo que luego se conoció como estructura molecular). Kekule presentó "fórmulas gráficas" de algunas sustancias para que fuera mejor comprendida su propuesta.

Sin embargo, era poco lo que se podía avanzar sin conocer la estructura atómica. Fue luego de que los electrones fueron identificados que se empezó a pensar en el enlace químico de una manera diferente, en la que estos eran sus directos responsables. Fue el alemán Richard Abegg, quien en 1904 fue el primero en relacionar la valencia química con la distribución de los electrones en el átomo. Según él, cada elemento tiene una valencia (+) y una contravalencia (-), cuya suma siempre debe ser ocho, para parecerse a los átomos estables de los gases nobles. Notó que la valencia de un elemento correspondía al grupo en la tabla periódica. Abegg murió en 1910 en un accidente de globo y no vió cómo sus ideas fueron desarrollándose. A partir de este momento, se entiende por valencia de un átomo el número de electrones de su capa más externa. Paul Drude sugirió que el número de valencia de Abegg era el número de electrones que tenía el átomo para enlazarse y el de contravalencia era el número de electrones de otros átomos que aquel podía remover o atraer más firmemente hacia si (Laidler, 1993).

En esta misma época, J.J. Thomson (1904) desarrolló su modelo atómico en el cual los electrones se encontraban distribuídos en capas o anillos concéntricos alrededor del centro de la esfera de carga positiva, y que únicamente los del anillo más externo estarían involucrados en un enlace; incluso, concluyó que debería haber una repetición periódica de las estructuras de los anillos más externos y que los gases nobles deberían tener este anillo completo. Este modelo y lo que implicaba, permitió un gran avance en las ideas respecto del enlace químico.

En 1913 fue adjudicado el Premio Nobel de Química al alemán Alfred Werner (1913) por su trabajo sobre la unión de los átomos para formar moléculas, por considerarse que daría nuevas luces sobre las anteriores investigaciones y que abriría nuevos campos de investigación, especialmente en la química inorgánica. Toda la teoría química moderna estaba basada en el concepto de valencia, que Werner concibió de una manera diferente a la convencional. Se asumía que las fuerzas de enlace de un átomo brotaban del centro del átomo, atrayendo uniformemente en todas las direcciones, y se expresaban con un número dado de líneas, igual a la valencia; Werner asumió que las fuerzas de enlace estaban distribuidas en una superficie esférica. Diferenció entre valencias primarias y secundarias, siendo las primeras las que tienen los átomos o grupos de átomos que se presentan como iones, y las segundas aquellas cuyo contenido de energía es menor que el de las primarias, pero que no difieren mucho de ellas, que producen complejos atómicos que no presentan iones independientes, como el agua o el amoniaco. Werner llamó número de coordinación al número de átomos o grupo de estos que se pueden unir en una primera esfera con un átomo de un elemento que funciona como centro.

El físico alemán Walter Kossel (1916) desarrolló una teoría del enlace, basada en la teoría de Abegg, introduciendo el concepto de electrovalencia, que explica la transferencia completa de electrones desde un átomo hasta otro para formar iones con estructura electrónica de gas noble, que se unen luego por atracciones electrostáticas. La aplicó exitosamente a enlaces polares, pero tuvo problemas cuando la quiso aplicar a enlaces no polares, por lo que vió que la teoría requería modificaciones. Su mayor acierto consistió en asegurar que todos los átomos al enlazarse buscaban tener estructura electrónica de gases nobles. 
Por la misma época, Gilbert N. Lewis (1916) estableció la teoría del enlace químico por compartición de pares de electrones y explicó que la diferencia esencial entre una molécula polar y otra no polar es que, en la primera, uno o más electrones son atraídos con suficiente fuerza para lograr que se desplacen de su lugar original en el átomo, produciéndose en la molécula un dipolo de alto momento eléctrico y llegando, en un caso extremo, a pasar completamente al otro átomo, caso en el cual se forman iones; mientras que en una molécula no polar los electrones permanecen en su posición original, igualmente compartidos por los dos átomos. Es importante comentar que, tres años antes de la publicación del artículo que se mencionó, Lewis (1913) en otro artículo propuso que se clasificaran los compuestos químicos como polares y no polares, en vez de como inorgánicos y orgánicos, aduciendo que las dos clasificaciones estaban relacionadas, ya que los compuestos orgánicos son no polares, mientras que la mayoría de los inorgánicos son más o menos polares. Sin embargo, aclaró que existen compuestos inorgánicos no polares y compuestos orgánicos con cierta parte de la molécula fuertemente polar.

En su artículo de 1916, Lewis dio a conocer su teoría de la estructura atómica externa, la teoría del cubo, en la cual representó los electrones de la capa más externa del átomo, que debían ser máximo ocho (haciendo alusión a la ley de valencia de Abegg), en las aristas de un cubo. Con este modelo pudo representar átomos de diferentes elementos y, también, moléculas sencillas, en las cuales dos cubos compartían uno o más electrones. No tuvo problema para explicar la diferencia entre los compuestos polares y no polares, con su modelo de los cubos: Los enlaces en los cuales un electrón puede ser propiedad común de las capas atómicas de dos átomos diferentes (lo que luego se conoció como enlace covalente) y los compuestos muy polares, en los cuales un átomo tiende a ceder los electrones, formando iones positivos, mientras que el otro átomo recibe los electrones, formando iones negativos. Explicó, luego, la atracción eléctrica de los iones de carga contraria para formar sólidos. Pudo explicar el enlace doble, pero le quedó imposible explicar el triple. Aseguró que esta propuesta la tenía desde 1902, no con el ánimo de reclamar prioridad sobre otras teorías, sino para reforzar que la suya fue desarrollada independientemente de esas otras.

Lewis (1916) utilizó el concepto de kernel, significando con este el número de cargas positivas en el núcleo del átomo que neutralicen las cargas de los electrones de la última capa. Postuló que un grupo de ocho electrones en la capa más externa es muy estable; en el caso del átomo de neón, con un kernel de ocho cargas positivas, tiene ocho electrones en la capa externa, mientras que un ión fluoruro, con ocho electrones en la capa externa (-8), tiene un kernel de +7 , lo que hace que se le asigne un "número polar" de -1 . El ión $\mathrm{Na}^{+}$, sin electrones en su capa externa y un kernel de +1 , tiene un número polar de +1 . Propuso usar los símbolos del kernel, en vez del símbolo ordinario del elemento, rodeando cada símbolo con un número de puntos igual a la valencia del átomo. Así pudo escribir las fórmulas estructurales de la siguiente manera: H:H, H:Ö:H, :I:Ï: .

Irving Langmuir (1919 a; b) propuso una teoría, a la que él mismo consideró como una extensión de la teoría del átomo cúbico de Lewis, y la llamó Teoría del Octeto de Valencia, expresada en términos de la siguiente ecuación: e $=8 \mathrm{n}-2 \mathrm{p}$, en la cual e es el número total de valencias (electrones en la capa externa) de los átomos de la molécula, n es el número de octetos y p el número de pares de electrones comprometidos en un enlace. Propuso que se definiera valencia como el número de pares de electrones que un átomo dado comparte con otros; y en vista de que la palabra valencia se usaba para expresar cosas diferentes, recomendó usar la palabra covalencia, origen del término enlace covalente.

Los físicos se mantuvieron mucho tiempo sin reconocer la importancia de la teoría del enlace de Lewis objetando, principalmente, el que Lewis parecía limitar a los electrones a 
una posición fija, además de que el par de electrones compartido, según ellos, no tendría suficiente fuerza para mantener unidos los átomos. Sin embargo, cuando Lewis publicó su libro "Valence and the Structure of Atoms and Molecules", en 1923, mostró ideas completamente consistentes con las de los físicos (Laidler, 1993).

Todas las teorías desarrolladas alrededor del concepto de valencia, hasta este momento, no tenían en cuenta los principios de la mecánica cuántica. Consideraban el átomo tal como lo describió Bohr en su modelo (1913), en el cual los electrones giraban alrededor del núcleo en diferentes órbitas. A raiz de la publicación de la ecuación de Schrödinger, se aplicó esta a los electrones implicados en la formación de enlaces covalentes para calcular las estructuras más estables. La palabra órbita vino a ser remplazada por la de orbital, para referirse a una función de onda que relacionara a un electrón simple con un átomo o una molécula. Este cambio era indispensable, puesto que, desde la mecánica cuántica, se vio que era imposible conocer el recorrido de un electrón alrededor del núcleo que permitiera especificar una órbita.

La molécula de $\mathrm{H}_{2}$, que se puede considerar como el prototipo del enlace totalmente no polar, se convirtió en el objeto de investigación desde las teorías de la mecánica cuántica. Los físicos alemanes Walter Heitler y Fritz London, en 1927, publicaron un artículo resultante de sus investigaciones, en el cual desarrollaron la que después llamaron teoría del enlace de valencia. En este, construyeron una función de onda para la molécula de hidrógeno, desde las funciones de onda de los dos átomos separados. Encontraron que la energía relacionada con un enlace covalente es de dos tipos: energía culómbica y energía de intercambio.

El químico estadounidense Linus Pauling (1927) continuó trabajando con la teoría de Heitler y London, conciente de la importancia de la formulación de Schrödinger para la química. Comparó las distancias entre los iones calculadas desde las teorías de la mecánica cuántica con las determinadas mediante la aplicación de rayos X sobre los cristales iónicos, y vió que coincidían. También trabajó sobre la molécula de hidrógeno y publicó dos artículos sobre su trabajo (Pauling, 1928 a; b). Publicó una serie de siete artículos llamada "The Nature of Chemical Bond", que introduce nuevos conceptos como el de resonancia, electronegatividad relativa, enlace iónico y enlace covalente, y del cual se publicó un capítulo en el Journal of American Chemical Society (Pauling, 1932). Sobre el mismo tema, y junto con otros científicos, publicó, más adelante, otros artículos relacionados con el enlace químico y las estructuras de los compuestos, proponiendo la teoría del orbital molecular para explicar los enlaces. Por la cantidad y calidad de los trabajos de Linus Pauling en este campo del saber, se le reconoce el haber transformado el campo de la química al introducir las teorías de la mecánica cuántica en el concepto de enlace químico, aunque no fue el primero en tener este interés.

\section{El concepto de valencia en los libros de texto}

Como se ha considerado anteriormente, la enseñanza de las ciencias está relacionada con las concepciones epistemológicas, pedagógicas y didácticas que ha elaborado el profesor. De igual forma, los libros de texto como material didáctico que son, responden implícita o explícitamente a unas concepciones sobre la ciencia y sobre la actividad científica, en los cuales, sus autores emplean generalmente versiones simplificadas de ella (Quílez y Sanjosé, 1996). Los trabajos correspondientes al análisis de textos, dada su complejidad y extensión se centran en núcleos temáticos o tópicos concretos, cuya selección responde a un interés educativo (González, García y Martínez, 2003). Para el presente trabajo, en el que ha quedado manifiesto el interés por la inserción de la historia y la filosofía de la ciencia en la enseñanza de ésta (Quílez y Sanjosé, 1996; Páez, Rodríguez y Niaz, 2002; Furió y Guisasola, 1997; Lombardi, 
1997; Izquierdo, 1988; Solves y Traver, 1996, 2001; Paruelo, 2003, Furió, Azcona y Guisasola, 1999; y Muñoz y Bertomeu, 2003), se tuvo en cuenta lo referente al concepto de Valencia.

Como muestran las investigaciones en este campo, se pueden establecer las características fundamentales de la naturaleza de la ciencia y de la actividad científica que permiten detectar las visiones deformadas de éstas que se pueden dar en la enseñanza tradicional.

Para el propósito formulado, se ha diseñado un instrumento de tipo cualitativo para el análisis de textos, en el que se han establecido 12 criterios derivados del estudio históricoepistemológico del concepto de valencia, que dan cuenta de cómo es presentado este enfoque en el desarrollo de este concepto, del carácter progresivo del avance científico, de la imagen de la actividad científica y de la versión de ciencia que presentan.

En los textos analizados:

- Se reconoce que la elaboración del concepto de valencia ha sido una construcción en comunidad en la que han participado varios científicos, cada uno con aportes significativos para su época.

- Se presenta el concepto de valencia como una elaboración "terminada" en la que no se muestran las diferentes interpretaciones que se ha dado al concepto.

- Se menciona la ley de las proporciones múltiples de Dalton como la base para la construcción del concepto de valencia.

- Se hace referencia a los postulados de E. Frankland, quien se refería a Valencia bajo el nombre de "atomicidad", considerada ésta como la capacidad de combinación de los átomos de los elementos.

- Se menciona el carácter experimental en la contrastación de los postulados acerca de los números de atomicidad diferentes de Frankland.

- Se identifica la importancia que tuvo el desarrollo de la teoría atómica en la formulación del concepto de valencia química.

- Se define valencia de un átomo como el número de electrones presente en la última capa electrónica.

- Se reconoce el aporte de Alfred Werner (1913), Premio Nobel de Química, en la construcción del concepto de enlace químico relacionado con su propia concepción de valencia.

- Se menciona cómo Gilbert Lewis dio a conocer su teoría de la estructura atómica, en la que representó los electrones de la capa más externa del átomo, que debían ser máximo ocho.

- Se plantea cómo Gilbert Lewis estableció la teoría del enlace químico por compartición de pares de electrones.

- Se reconoce las limitaciones de Gilbert Lewis para explicar la formación de los enlaces triples en las moléculas.

- Se mencionan los aportes de I. Langmuir en la matematización de la forma de calcular el número de electrones de valencia presentes en la molécula.

- Se reconocen los aportes de las teorías de la mecánica cuántica en el desarrollo del concepto de valencia.

A partir de la aplicación del instrumento anterior a cada uno de los textos seleccionados se obtuvieron los resultados que se presentan en el siguiente cuadro: 
Rómulo Gallego Badillo, Royman Pérez Miranda, María V. Uribe Beltrán, Luigi Cuéllar Fernández y Rafael Y. Amador Rodríguez

Cuadro 1. Análisis de los textos de décimo grado $(n=6)$ y de educación superior $(n=3)$ utilizando los criterios derivados del estudio histórico-epistemológico del concepto de valencia.

\begin{tabular}{|c|c|c|c|c|c|c|c|c|c|c|c|c|c|c|}
\hline No & Textos & $\mathrm{C} 1$ & $\mathrm{C} 2$ & C3 & $\mathrm{C} 4$ & $\mathrm{C} 5$ & C6 & C7 & C8 & C9 & C10 & C11 & C12 & C13 \\
\hline 1 & $\begin{array}{l}\text { García, Aubad, } \\
\text { Zapata (1985) }\end{array}$ & $\mathrm{N}$ & M & $\mathrm{N}$ & $\mathrm{N}$ & $\mathrm{N}$ & $\mathrm{N}$ & M & $\mathrm{N}$ & $\mathrm{N}$ & $\mathrm{N}$ & $\mathrm{N}$ & $\mathrm{N}$ & $\mathrm{N}$ \\
\hline 2 & Manco (2001) & $\mathrm{N}$ & $\mathrm{M}$ & $\mathrm{N}$ & $\mathrm{N}$ & $\mathrm{N}$ & $\mathrm{N}$ & $\mathrm{M}$ & $\mathrm{N}$ & $\mathrm{N}$ & $\mathrm{N}$ & $\mathrm{N}$ & $\mathrm{N}$ & $\mathrm{N}$ \\
\hline 3 & Poveda (1996) & $\mathrm{N}$ & $\mathrm{M}$ & $\mathrm{N}$ & $\mathrm{N}$ & $\mathrm{N}$ & $\mathrm{N}$ & $\mathrm{M}$ & $\mathrm{N}$ & $\mathrm{N}$ & M & $\mathrm{N}$ & $\mathrm{N}$ & $\mathrm{N}$ \\
\hline 4 & $\begin{array}{l}\text { Mondragón, } \\
\text { Peña, Sánchez y } \\
\text { Fernández } \\
(2001)\end{array}$ & $\mathrm{N}$ & M & $\mathrm{N}$ & $\mathrm{N}$ & $\mathrm{N}$ & $\mathrm{N}$ & M & $\mathrm{N}$ & $\mathrm{N}$ & M & $\mathrm{N}$ & $\mathrm{N}$ & $\mathrm{N}$ \\
\hline 5 & $\begin{array}{l}\text { Castelblanco, } \\
\text { Sánchez, Peña } \\
(2003)\end{array}$ & $\mathrm{N}$ & M & $\mathrm{N}$ & $\mathrm{N}$ & $\mathrm{N}$ & $\mathrm{N}$ & M & $\mathrm{N}$ & $\mathrm{N}$ & $\mathrm{N}$ & $\mathrm{N}$ & $\mathrm{N}$ & $\mathrm{N}$ \\
\hline 6 & $\begin{array}{l}\text { Cárdenas y } \\
\text { Gélves (1999) }\end{array}$ & $\mathrm{N}$ & M & $\mathrm{N}$ & $\mathrm{N}$ & $\mathrm{N}$ & $\mathrm{N}$ & M & $\mathrm{N}$ & $\mathrm{N}$ & M & $\mathrm{N}$ & $\mathrm{N}$ & $\mathrm{N}$ \\
\hline $7^{*}$ & Chang (1999) & $\mathrm{N}$ & $\mathrm{N}$ & $\mathrm{N}$ & $\mathrm{N}$ & $\mathrm{N}$ & $\mathrm{M}$ & $\mathrm{M}$ & $\mathrm{C}$ & $\mathrm{N}$ & $\mathrm{M}$ & $\mathrm{N}$ & $\mathrm{N}$ & $\mathrm{N}$ \\
\hline $8^{*}$ & Petrucci (1999) & M & M & $\mathrm{N}$ & $\mathrm{N}$ & $\mathrm{N}$ & $\mathrm{N}$ & M & $\mathrm{N}$ & $\mathrm{N}$ & M & M & $\mathrm{N}$ & $\mathrm{N}$ \\
\hline $9^{*}$ & Daub (1996) & $\mathrm{N}$ & $\mathrm{M}$ & $\mathrm{N}$ & $\mathrm{N}$ & $\mathrm{N}$ & $\mathrm{N}$ & $\mathrm{M}$ & $\mathrm{N}$ & $\mathrm{N}$ & $\mathrm{M}$ & $\mathrm{N}$ & $\mathrm{N}$ & $\mathrm{N}$ \\
\hline
\end{tabular}

C1 al C13 : Criterios derivados del estudio histórico-epistemológico del concepto de valencia.

* Textos utilizados en educación superior.

$$
\begin{gathered}
\mathrm{M}=\text { Menciona } \\
\mathrm{N}=\text { No menciona } \\
\mathrm{C}=\text { Presenta el tema en el contexto histórico-epistemológico }
\end{gathered}
$$

\section{Conclusiones e implicaciones didáticos}

El conocer bien la ciencia a enseñar, no solamente implica comprender los modelos, teorías o conceptos científicos que se hacen objeto de trabajo en el aula, sino que, además, se ha de conocer el contexto histórico dentro del cual se han construido tales modelos, teorías o conceptos, con el fin de elaborar una interpretación de su historia interna y externa, con el propósito de presentar una versión de ciencia dinámica, producto del trabajo en comunidad.

La revisión histórica y epistemológica del concepto de valencia realizada permite establecer que desde la formulación de las leyes de las proporciones definidas y múltiples hasta finales del siglo XIX, debido al desconocimiento de la estructura atómica, los científicos formularon versiones similares que intentaban explicar la capacidad combinatoria de los átomos de los elementos, comprobándola experimentalmente, sin poder avanzar en el desarrollo del conocimiento sobre el enlace químico. A partir de la formulación de los modelos atómicos en los cuales los electrones se distribuían en capas alrededor del núcleo, los científicos propusieron modelos y teorías que relacionaban la capacidad combinatoria de los átomos (valencia) con los electrones de la capa más externa del átomo (electrones de valencia).

Con la inclusión de la historia de las ciencias en su enseñanza se puede establecer un hilo conductor que da cuenta del progreso científico y, a su vez, identificar los conceptos estructurantes 
que permiten la introducción de otros conocimientos para, de esta forma, proponer soluciones ante los obstáculos epistemológicos (Solbes y Traver, 1996). En el caso del concepto de valencia, se pudo establecer la relevancia de los modelos atómicos para desarrollar las teorías del enlace químico, a partir del concepto de valencia, como capacidad combinatoria de los átomos, lo que vino a ser el concepto estructurante.

En lo referente al análisis de los textos, se establece que, tanto los de nivel de educación media como los de educación superior, presentan una definición del concepto de valencia que no tiene en cuenta la construcción histórica del concepto. Presentan el concepto de valencia referido a los electrones de la capa externa del átomo, sin encontrarse en ninguno de ellos una mención del concepto relacionada con el poder combinatorio de los átomos.

$\mathrm{Al}$ desconocer el aporte de cada uno de los científicos que contribuyeron en la construcción del concepto de valencia para llegar a formular las teorías del enlace químico, los textos analizados presentan una visión distorsionada de la actividad científica. Sin embargo, cabe reconocer que en los de educación superior hay un mayor reconocimiento de la importancia de la historia en la construcción del conocimiento en este campo, puesto que citan a algunos científicos, sin llegar a un acercamiento a la realidad del trabajo científico y al contexto en el que se desarrolla.

Los autores del presente artículo consideran que el involucrar a los estudiantes en el estudio histórico-epistemológico de un modelo, teoría o concepto científico, les puede permitir comprenderlos y hacerse una idea más aproximada del trabajo científico y del desarrollo de las ciencias, diferente de la habitual de ciencia absoluta, empirista y acumulativa.

\section{Referencias}

ADÚRIZ-BRAVO, A. Integración de la epistemología en la formación del profesorado de ciencias. 2001. Tesis (Doctorado en Didáctica de las Ciencias Experimentales)-Universidad Autónoma de Barcelona, Barcelona, 2001.

BACON, F. Novus organum. México: Editorial Porrúa, 2000. p. 35-218.

BOHR. N. On the constitution of atoms and molecules. Philosophical Magazine: serie 6, London, v. 26, p. 1-25, 1913.

FRANKLAND, E. On a new series of organic bodies containing metals. Philosophical Transactions of the Royal Society of London, London, v. 142, p. 417-444, 1852.

FURIÓ, C. Y.; GUISASOLA, J. Deficiencias epistemológicas en la enseñanza habitual de los conceptos de campo y potencial eléctrico. Enseñanza de las Ciencias, Barcelona, v. 15 n. 2, p. 259-271, 1997.

FURIÓ, C.; AZCONA, R. Y.; GUISASOLA, J. Dificultades conceptuales y epistemológicas del profesorado en la enseñanza de los conceptos de cantidad de sustancia y mol. Enseñanza de las Ciencias, Barcelona, v. 17, n. 3, p. 359-376, 1999.

GALLEGO B., R.; PÉREZ, M., R. El problema del cambio en las concepciones epistemológicas, pedagógicas y didácticas. Bogotá: Universidad Pedagógica Nacional, 1999. 
Rómulo Gallego Badillo, Royman Pérez Miranda, María V. Uribe Beltrán, Luigi Cuéllar Fernández y Rafael Y. Amador Rodríguez

GALLEGO, R. et al. La formación inicial de profesores de ciencias en Colombia contrastación de los fundamentos. Bogotá: Universidad Pedagógica Nacional, 2004.

GIL, D. ¿Qué hemos de saber y saber hacer hacer los profesores de ciencias? Eneñanza de las Ciencias, Barcelona, v. 9, n. 1, p. 69-77, 1991.

GONZÁLEZ, C.; GARCÍA, S.; MARTÍNEZ, L. ¿A qué contenidos relacionados con la fotosíntesis dan más importancia los textos escolares de secundaria? Enseñanza de las Ciencias, Barcelona, p. 77-88, 2003. Número Extra.

HODSON, D. Phylosophy of science, science and science education. Studies in Science Education, Leeds, v.12, n. 1, p. 25-27, 1985.

ISLAS, S. M.; PESA, M. A. ¿Qué rol asignan los profesores de física de nivel medio a los modelos científicos y a las actividades de modelado? Enseñanza de las Ciencias, Barcelona, p. 57-66, 2003. Número Extra.

IZQUIERDO, M. La contribución de la teoría del flogisto a la estructuración actual de la ciencia química: implicaciones didácticas. Enseñanza de las Ciencias, Barcelona, v. 6, n. 1, p. 67-74, 1988.

KEKULE, F. Studies on aromatic compounds. Annalen der Chemie Und Pharmacial, Leipzig v. 137, p. 129-196, 1865.

KOSSEL, W. Molecule formation as a question of atomic structure. Annalen der Physik, Leipzig, v. 49, p. 229-362, 1916.

KUHN, T. S. La estructura de las revoluciones científicas. Traducido por A. Contín. México: Fondo de Cultura Económica, 1962.

LAIDLER, K. J. The world of physical chemistry. New York: Oxford University Press, 1993. p. 339354.

LANGMUIR, I. The structure of atoms and the octet theory of Valence. Proceedings of the National Academy of Science, Allahabad, v. 252, 1919a.

. The arrangement of electrons in atoms and molecules. Journal of the American Chemical Society, Easton, v. 41, n. 6, p. 868, 1919 b.

LEWIS, G. Valence and tautomerism. Journal of the American Chemical Society, Easton, v. 35, p. 1448, 1913.

LEWIS, G. The atom and the molecule. Journal of the American Chemical Society, Easton, v. 38, p. $762,1916$.

LOMBARDI, O. I. La pertinencia de la historia en la enseñanza de ciencias: argumentos y contraargumentos. Enseñanza de las Ciencias, Barcelona, v. 15, n. 3, p. 343-349, 1997. 
MUNOOZ, R.; BERTOMEU, J. La historia de la ciencia en los libros de texto: la(s) hipótesis de avogadro. Enseñanza de las Ciencias, Barcelona, v. 21, n. 1, p. 147-159, 2003.

PÁEZ, Y.; RODRÍGUEZ, M.; NIAZ, M. La teoría atómica de Dalton desde la perspectiva de la nueva filosofía de la ciencia: Un análisis de la imagen reflejada por los textos de química de bachillerato. Revista Paradigma, Maracay, v. 23, n. 2, p. 97-122, 2002.

PARUELO, J. Enseñanza de las ciencias y filosofía. Enseñanza de las Ciencias, Barcelona, v. 21, n. 2, p. 329-335, 2003.

PAULING, L. The size of ions and the structure of ionic crystals. Journal of American Chemical Society, Easton, v. 49, p. 765-790, 1927.

. The shared-electron chemical bond. Proceedings of the National Academy of Science, Allahabad, v. 14, p. 359-362, 1928.

. The application of the quantum mechanics to the structure of the hidrogen molecule and hidrogen molecule ion and to related problems. Chemical Reviews, Baltimore, v. 5, p.173-213, 1928.

. The nature of the chemical bond: the energy of single bonds and the relative electronegativity of atoms. Journal of the American Chemical Society, Easton, v. 54, p. 3570, 1932.

POPPER, K. La lógica de la investigación científica. Madrid: Ediciones Tecnos, 1962.

QUÍLEZ, J.; SANJOSÉ, V. El principio de Le Chatelier a través de la historia y su formulación didáctica en la enseñanza del equilibrio químico. Enseñanza de la Ciencias, Barcelona, v. 14, n. 3, p. 381-390, 1996.

SOLBES, J.; TRAVER, M. J. La utilización de la historia de las ciencias en la enseñanza de la física y la química. Enseñanza de las Ciencias, Barcelona, v. 14, n. 1, p. 103-112, 1996.

- Resultados obtenidos introduciendo historia de las ciencias en las clases de física y química: mejora de la imagen de la ciencia y desarrollo de actitudes positivas. Enseñanza de las Ciencias, Barcelona, v. 19, n. 1, p. 151-162, 2001.

THOMSON, J. J. On the structure of the atom. Philosophical Magazine: series 6, London, v. 7, n. 39, p. 237-265, 1904.

THOMSON, T. On the daltonian theory of definite proportions in chemical combinations. Annals of Philosophy, v. 2, p. 32, 1913.

WERNER, A. On the constitution and configuration of higer-order compounds: nobel lectures, chemistry 1901-1921. Amsterdam: Elsevier Publishing Company, 1913. 